\title{
Mitigazione dei cambiamenti climatici con tecnologie biologiche di cattura e recupero delle $\mathrm{CO} 2$
}

\author{
MARCO GIORDANO ${ }^{1}$
}

${ }^{1}$ Affiliation not available

\begin{abstract}
In questo articolo vengono riportati alcuni aspetti relativi alla cattura ed all'utilizzo della anidride carbonica, mediante applicazione delle biotecnologie basate sulle microalghe. In particolare vengono riportati i principali sistemi impiegati per la coltivazione di questi organismi fotoautotrofi ed i più rilevanti parametri di progettazione.
\end{abstract}

\section{Introduzione}

Le crescenti emissioni in atmosfera di gas serra $(\mathrm{GHG})$ da parte delle attività antropiche, rappresentano la principale causa dei cambiamenti climatici ${ }^{1,2,3}$. Tra questi GHG, il principale è l'anidride carbonica $\left(\mathrm{CO}_{2}\right)$. Diverse azioni e misure nazionali ed internazionali sono state identificate ${ }^{4}$ al fine di tentare di ridurre le emissioni di $\mathrm{CO}_{2}$ in atmosfera; non ultime quelle identificate nella più recente Conferenza svolta a Madrid nel dicembre del $2019^{5}$. Accanto alla necessità di diminuzione delle emissioni in atmosfera, nella letteratura tecnico scientifica di settore sono ormai note anche altre possibilità di operare, attraverso azioni di cattura e riutilizzo. Difatti le azioni di cattura, stoccaggio ed utilizzo dell' anidride carbonica (CCS e CCU) sono indicate come uno dei metodi più efficaci da perseguire, ove non è possibile operare una riduzione delle emissioni alla fonte. Il 
presente lavoro si inserisce nell'ambito dello studio delle tecnologie innovative basate sulle alghe per la cattura ed il riutilizzo della $\mathrm{CO}_{2}$.

\section{CATTURA, STOCCAGGIO E RIUTILIZZO DELLA $\mathrm{CO}_{2}$}

I processi industriali, le centrali termoelettriche e la deforestazione oltre ad altri, hanno portato a un aumento incessante delle emissioni di gas serra $(\mathrm{GHG})^{6,7,8,9} . \mathrm{La} \mathrm{CO}_{2}$ presente in atmosfera, infatti, ha raggiunto un livello allarmante con previsione di un ulteriore crescita ${ }^{10}$.Diverse opzioni potrebbero aiutare a raggiungere l'obiettivo di mitigazione dei cambiamenti climatici, tra cui la cattura e lo stoccaggio del carbonio (CCS) e la cattura e utilizzo del carbonio (CCU) ${ }^{11}$, che permettono di catturare le emissioni puntuali di $\mathrm{CO}_{2}$ limitandone il rilascio nell'atmosfera. La differenza tra CCS e CCU consiste nella destinazione finale della $\mathrm{CO}_{2}$ catturata, infatti con la CCS, viene trasferita in un sito adatto e sicuro per l'archiviazione a lungo termine, mentre con CCU, viene convertita in prodotti commerciali. Tra le opzioni di cattura della $\mathrm{CO}_{2}$ troviamo la fissazione mediante microalghe, considerata un applicazione di CCU piuttosto che di CCS, poiché le microalghe non vengono coltivate semplicemente per catturare $\mathrm{CO}_{2}$, ma soprattutto per la possibilità di essere trasformate in bioprodotti come biocombustibili ${ }^{11}$. Le microalghe rappresentano una promettente fonte di produzione di energia sostenibile, che è particolarmente importante nel contesto odierno in cui i combustibili fossili si stanno esaurendo rapidamente. Rispetto ai metodi di CCS, le microalghe comportano molti vantaggi, come: un alto tasso di fotosintesi, un rapido tasso di crescita, un'alta compatibilità ambiente e minori costi di applicazione.

Le alghe sono organismi fotoautotrofi, che a partire da $\mathrm{CO}_{2}$ ed energia solale, producono ${ }^{12}$ :

- molecole complesse per il proprio metabolismo energetico e biosintetico;

- ossigeno che in parte usano per la respirazione ed in parte rilasciano nel mezzo esterno.

Le variazioni di dimensione nelle specie di alghe sono davvero sorprendenti e le distinguiamo in 
due principali categorie: macroalghe e microalghe. Diversi ceppi di alghe possono prosperare in acqua dolce o salata e sono in grado di sopravvivere in condizioni differenti di temperatura, $\mathrm{pH}$, torbidità, concentrazioni di $\mathrm{O}_{2}$ e $\mathrm{CO}_{2}$ ecc.). Le microalghe sono caratterizzate da varia quantità e tipologia di pigmenti fotosintetici (soprattutto clorofilla e carotenoidi) che ne permette una distinzione in: cianobatteri blu-verdi (Cyanophyta) per la presenza di ficocianina, alghe rosse (Rhodophyta) per la ficoeritrina, le verdi (Chlorophyta) per la clorofilla a \& b, le brune (Phaeophyceae) e Bacillariophyta/diatomee per le xantofille (come fucoxantine) e caroteni ${ }^{13}$. Tra i principali parametri monitorati per controllare e migliorare la crescita algale vi è la temperatura. La maggior parte sono organismi mesofili, con un ottimo di crescita nell' intervallo compreso tra $20^{\circ} \mathrm{C}$ e $30^{\circ} \mathrm{C}$, altre si sono adattate alle basse temperature, con un metabolismo attivo e crescita fra $0^{\circ} \mathrm{C}$ e $12^{\circ} \mathrm{C}$ e sono definite psicrofile mentre quelle capaci di svilupparsi ad elevati valori di temperatura, fra i $35^{\circ} \mathrm{C}$ ed i $60^{\circ} \mathrm{C}$ sono dette termofile ${ }^{14}$. In generale, i sistemi di coltivazione delle alghe sono suddivisi in due macrocategorie principali: sistemi aperti o sistemi chiusi (fotobioreattore) ${ }^{15}$.

I sistemi aperti sono ampiamente utilizzati grazie ai bassi costi operativi/costruzione ed alla capacità di assorbire la $\mathrm{CO}_{2}$ direttamente dall' atmosfera ambientale ${ }^{16}$ ma sono caratterizzati da una bassa concentrazione di biomassa microalgale.

I sistemi chiusi (fotobioreattori) permettono, invece, una coltivazione ad alta concentrazione di biomassa, di controllare efficacemente le condizioni di crescita e garantiscono un facile controllo di eventuali contaminazioni ${ }^{17}$. 


\section{PRINCIPALI FATTORI NELLA PROGETTAZIONE DI UN FOTOBIOREATTORE}

In condizioni naturali, le alghe assorbono la luce solare, assimilano l'anidride carbonica dall'aria e le sostanze nutritive dagli habitat acquatici. Pertanto, la produzione artificiale dovrebbe replicare e migliorare le condizioni di crescita naturali. Nella progettazione di un fotobioreattore, la penetrazione, la cattura e la distribuzione della luce sono i fattori dominanti. Inoltre, una buona miscelazione, nonché temperatura e $\mathrm{pH}$ favorevoli possono migliorare significativamente la crescita delle microalghe ${ }^{18}$.

La luce naturale viene spesso impiegata per la coltivazione delle microalghe, con conseguente notevole riduzione dei costi. Tuttavia, la luce solare muta inevitabilmente a causa delle condizioni metereologiche, dei cicli giornalieri e delle stagioni, rendendo tale applicazione redditiva per la produzione commerciale solo nelle aree con un'elevata radiazione solare. Per superare tali limiti vengono impiegati anche mezzi artificiali come lampade fluorescenti. L'illuminazione artificiale permette una produzione continua, ma con un input energetico significativamente più elevato. Spesso la fornitura di elettricità per l'illuminazione artificiale è derivata da combustibili fossili annullando l'obiettivo primario di sviluppare un biocombustibile competitivo in termini di prezzo e con bassa impronta di carbonio dei sistemi. La distribuzione della luce non è uniforme all'interno di un PBR bensì subisce un'attenuazione in funzione della lunghezza d'onda della luce, dalla con-

centrazione cellulare, dalla geometria del PBR e dalla distanza di penetrazione della luce ${ }^{19}$. È noto che l'intensità della luce diminuisce al crescere dello spessore del PBR. Se l'intensità della luce raggiunge il livello di saturazione, la crescita è inibita dalla luce (fotoinibizione), mentre se l'intensità della luce è al di sotto del livello necessario per attivare la fotosintesi, la crescita è limitata dalla luce (fotolimitazione).

La miscelazione previene e limita la sedimentazione cellulare, la formazione di zone morte e 
l'attaccamento cellulare alle pareti del PBR. Inoltre, la miscelazione garantisce che tutte le cellule siano ugualmente esposte alla luce e promuove il trasferimento di massa. Tuttavia, un'eccessiva miscelazione può danneggiare le cellule e provocare il collasso della coltura, a causa delle forze di taglio generate ${ }^{20}$. La miscelazione garantisce anche un ciclo luce/buio periodico, dove le microalghe si spostano tra la zona illuminata (vicino alla superficie del PBR) e la zona scura (al centro del PBR), promuovendo la conversione fotosintetica ${ }^{21}$.

Il $\mathrm{pH}$ è un parametro fondamentale per la crescita della biomassa algale. La maggior parte delle specie di microalghe ha un intervallo di $\mathrm{pH}$ preferito tra 7 e 9 . Tuttavia, alcune specie possono tollerare intervalli più acidi o basici ${ }^{22}$. È fondamentale mantenere il $\mathrm{pH}$ della coltura entro il valore ottimale per evitare che la coltura subisca danneggiamenti.

La temperatura durante la coltivazione può subire un notevole cambiamento a causa delle variazioni giornaliere e stagionali, pertanto, un sistema di controllo della temperatura affidabile ed economico è essenziale ${ }^{23}$. Per il controllo della temperatura possono essere utilizzati teli ombreggianti ${ }^{24}$, irrigazione con acqua quando la temperatura della coltura è elevata, l'immersione totale o parziale del PBR in piscine d'acqua ${ }^{25}$ e l'installazione di scambiatori di calore connessi al PBR. I sistemi di controllo della temperatura incrementano i costi di coltivazione ed il metodo più efficace ed economico consiste nello spruzzo di acqua solo durante il surriscaldamento per poi riutilizzarla dopo il raffreddamento.

Il Controllo delle specie contaminanti e pulizia del sistema rappresentano altri due aspetti fondamentali. Si può tollerare un certo grado di impurità nelle colture di microalghe quando i prodotti generati sono di basso valore come i biocarburanti, ma bisogna evitare una contaminazione eccessiva per garantire una stabile qualità dei prodotti. La pulizia del PBR è di fondamentale importanza perché previene la formazione di biofilm sulla parete mantenendo un'elevata penetrazione della luce $^{22,26}$. 


\section{CONCLUSIONI}

Il riscaldamento globale, l'aumento delle emissioni di $\mathrm{CO}_{2}$, il consumo di combustibili fossili ed il loro esaurimento sono fattori che negli ultimi anni spingono la ricerca a sviluppare fonti energetiche alternative, rinnovabili e pulite ${ }^{9,7}$. In questo contesto, la biomassa algale sta diventando un'importante risorsa dalla quale è possibile estrarre biocombustibili. Alcuni studi presenti in letteratura riportano applicazioni delle tecnologie a base di alghe e testimoniano l'influenza della specie di microalga, della tipologia di sistema di coltivazione, del $\mathrm{pH}$, della temperatura e della densità di biomassa iniziale sulle efficienze di rimozione della $\mathrm{CO}_{2}$. La coltivazione di microalghe viene effettuata in sistemi aperti e sistemi chiusi. I primi sono maggiormente utilizzati e comportano bassi costi di costruzione/gestione, facilità di esecuzione/manutenzione ma soffrono la scarsa penetrazione della luce, la contaminazione e la limitata produttività della biomassa. I secondi includono diverse configurazioni di fotobioreattori (PBR) sono più costosi e generalmente comportano un migliore trasferimento di massa, una biomassa di alta qualità, una maggiore produttività, ridotti consumi di acqua e un controllo efficace dei parametri di processo, $\mathrm{CO}_{2}$, OD ed eventuali forme di contaminazione. La crescita di microrganismi fotosintetici è un processo influenzato da molti fattori ambientali come luce, miscelazione, trasferimento di massa, temperatura e $\mathrm{pH}$ che necessitano di ulteriori studi per rendere la tecnologia applicabile a grande scala.

\section{References}

1.Zarra, T., Giuliani, S., Naddeo, V. \& Belgiorno, V. Control of odour emission in wastewater treatment plants by direct and undirected measurement of odour emission capacity. Water Science and Technology 66, 1627-1633 (2012).

2.Zarra, T., Galang, M. G., Ballesteros, F., Belgiorno, V. \& Naddeo, V. Environmental odour management by artificial neural network - A review. Environment International 133, 105189 
(2019).

3.Ensano, B. M. B., Borea, L., Naddeo, V., Luna, M. D. G. de \& Belgiorno, V. Control of emerging contaminants by the combination of electrochemical processes and membrane bioreactors. Environmental Science and Pollution Research 26, 1103-1112 (2017).

4.Naddeo, V. \& Korshin, G. Water energy and waste: The great European deal for the environment. Science of The Total Environment 764, 142911 (2021).

5.Ensano, B. M. B. et al.. Applicability of the electrocoagulation process in treating real municipal wastewater containing pharmaceutical active compounds. Journal of Hazardous Materials 361, 367-373 (2019).

6.Belgiorno, V., Naddeo, V., Scannapieco, D., Zarra, T. \& Ricco, D. Ecological status of rivers in preserved areas: Effects of meteorological parameters. Ecological Engineering 53, 173-182 (2013).

7.Naddeo, V., Belgiorno, V., Zarra, T. \& Scannapieco, D. Dynamic and embedded evaluation procedure for strategic environmental assessment. Land Use Policy 31, 605-612 (2013).

8.Prado, M. et al.. Removal of emerging contaminant and fouling control in membrane bioreactors by combined ozonation and sonolysis. International Biodeterioration \& Biodegradation 119, 577-586 (2017).

9.Nikolaou, A. D. et al.. Multi-parametric water quality monitoring approach according to the WFD application in Evros trans-boundary river basin: priority pollutants. Desalination 226, 306-320 (2008).

10.Yadav, G., Karemore, A., Dash, S. K. \& Sen, R. Performance evaluation of a green process for microalgal $\mathrm{CO} 2$ sequestration in closed photobioreactor using flue gas generated in-situ. Bioresource Technology 191, 399-406 (2015). 
11.Cuéllar-Franca, R. M. \& Azapagic, A. Carbon capture storage and utilisation technologies: A critical analysis and comparison of their life cycle environmental impacts. Journal of CO2 Utilization 9, 82-102 (2015).

12.Zhao, B. \& Su, Y. Process effect of microalgal-carbon dioxide fixation and biomass production: A review. Renewable and Sustainable Energy Reviews 31, 121-132 (2014).

13.Cheah, W. Y., Show, P. L., Chang, J.-S., Ling, T. C. \& Juan, J. C. Biosequestration of atmospheric CO 2 and flue gas-containing CO 2 by microalgae. Bioresource Technology 184, 190-201 (2015).

14.Rooke, J. C. et al.. Hybrid photosynthetic materials derived from microalgae Cyanidium caldarium encapsulated within silica gel. Journal of Colloid and Interface Science 344, 348-352 (2010).

15.McGinn, P. J. et al.. Integration of microalgae cultivation with industrial waste remediation for biofuel and bioenergy production: opportunities and limitations. Photosynthesis Research 109, 231-247 (2011).

16.Wu, W., Wang, P.-H., Lee, D.-J. \& Chang, J.-S. Global optimization of microalgae-to-biodiesel chains with integrated cogasification combined cycle systems based on greenhouse gas emissions reductions. Applied Energy 197, 63-82 (2017).

17.Chisti, Y. Biodiesel from microalgae. Biotechnology Advances 25, 294-306 (2007).

18.Huang, Q., Jiang, F., Wang, L. \& Yang, C. Design of Photobioreactors for Mass Cultivation of Photosynthetic Organisms. Engineering 3, 318-329 (2017).

19.Quan, Y., Pehkonen, S. O. \& Ray, M. B. Evaluation of Three Different Lamp Emission Models Using Novel Application of Potassium Ferrioxalate Actinometry. Industrial \& Engineering Chemistry Research 43, 948-955 (2004). 
20.Fernández, F. G. A., Sevilla, J. M. F. \& Grima, E. M. Photobioreactors for the production of microalgae. Reviews in Environmental Science and Bio/Technology 12, 131-151 (2013).

21.Xue, S., Zhang, Q., Wu, X., Yan, C. \& Cong, W. A novel photobioreactor structure using optical fibers as inner light source to fulfill flashing light effects of microalgae. Bioresource Technology 138, 141-147 (2013).

22.Wang, M., Han, J., Dunn, J. B., Cai, H. \& Elgowainy, A. Well-to-wheels energy use and greenhouse gas emissions of ethanol from corn sugarcane and cellulosic biomass for US use. Environmental Research Letters 7, 045905 (2012).

23.Wang, B., Lan, C. Q. \& Horsman, M. Closed photobioreactors for production of microalgal biomasses. Biotechnology Advances 30, 904-912 (2012).

24.Ugwu, C. U., Aoyagi, H. \& Uchiyama, H. Photobioreactors for mass cultivation of algae. Bioresource Technology 99, 4021-4028 (2008).

25.Carlozzi, P., Pushparaj, B., Degl'Innocenti, A. \& Capperucci, A. Growth characteristics of Rhodopseudomonas palustris cultured outdoors in an underwater tubular photobioreactor, and investigation on photosynthetic efficiency. Applied Microbiology and Biotechnology 73, 789-795 (2006).

26.Borea, L. et al.. Wastewater treatment by membrane ultrafiltration enhanced with ultrasound: Effect of membrane flux and ultrasonic frequency. Ultrasonics 83, 42-47 (2018). 
Figure Captions

Figure 1. Fotobioreattore tubolare 
Figures

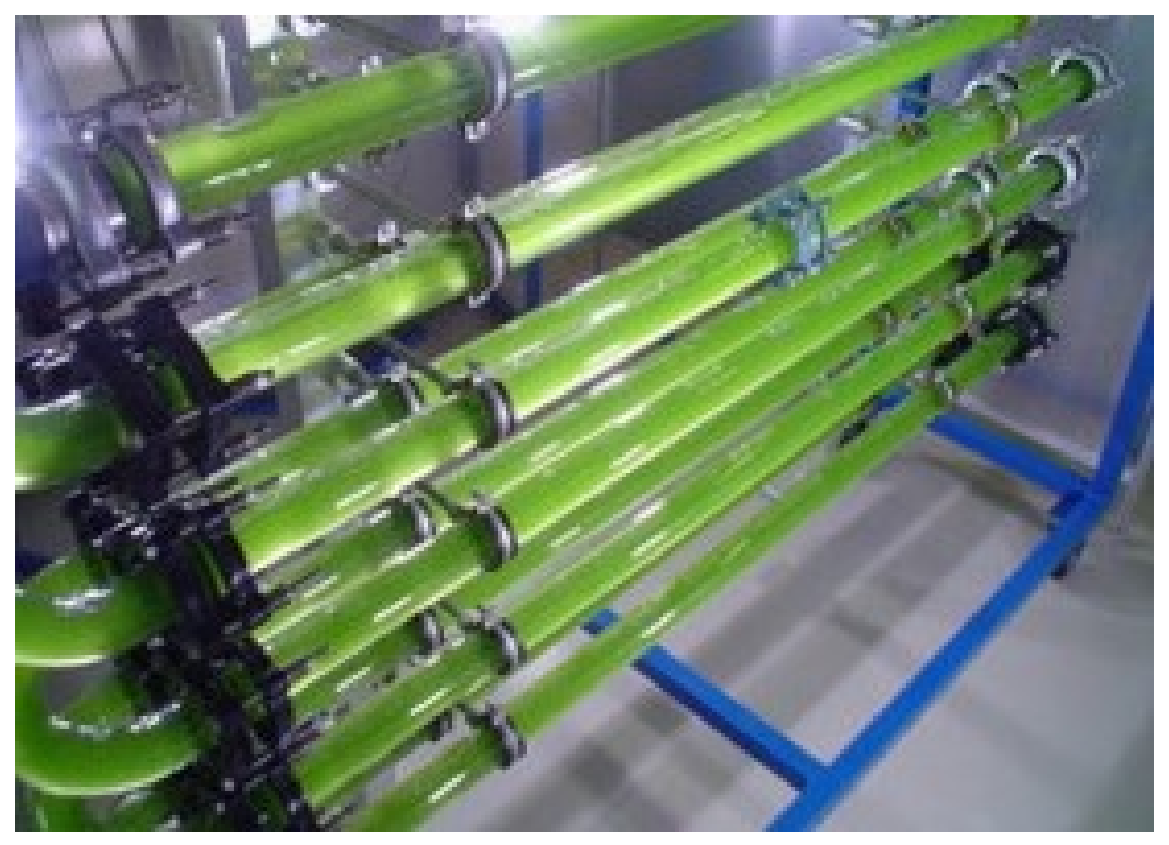

Figure 1: Fotobioreattore tubolare 